\title{
Meson properties in magnetized quark matter
}

\author{
Ziyue Wang and Pengfei Zhuang \\ Physics Department, Tsinghua University and Collaborative Innovation Center of Quantum Matter, \\ Beijing 100084, China
}

(Received 2 December 2017; published 23 February 2018)

\begin{abstract}
We study neutral and charged meson properties in the magnetic field. Taking the bosonization method in a two-flavor Nambu-Jona-Lasinio model, we derive effective meson Lagrangian density with minimal coupling to the magnetic field, by employing derivative expansion for both the meson fields and Schwinger phases. We extract from the effective Lagrangian density the meson curvature, pole and screening masses. As the only Goldstone mode, the neutral pion controls the thermodynamics of the system and propagates the long range quark interaction. The magnetic field breaks down the space symmetry, and the quark interaction region changes from a sphere in vacuum to a ellipsoid in magnetic field.
\end{abstract}

DOI: 10.1103/PhysRevD.97.034026

\section{INTRODUCTION}

A strong magnetic field may exist in the core of neutron stars and the beginning of nuclear collisions at high energies. The field strength in these cases is expected to reach $10^{19}$ Gauss, corresponding to $|e B| \sim 10 m_{\pi}^{2}$ [1-3]. In such an external magnetic field, the phase structure of a quantum chromodynamics (QCD) system will be significantly changed. For the phase transition from chiral symmetry breaking to its restoration, there are magnetic catalysis effects at the mean field level [4-6] and inverse magnetic catalysis effects in lattice QCD simulations [7-9] and effective model calculations [10-14]. Considering that pion mesons are the Goldstone modes corresponding to chiral symmetry breaking and dominate the QCD thermodynamics at low temperature, their properties [15-27] in an external magnetic field are extensively investigated.

In quark models mesons are considered as quantum fluctuations. They are usually constructed through nonperturbative methods like random phase approximation (RPA) $[16,18]$ and derivative expansion [28-30]. In the RPA approach, mesons are introduced via the summation of an infinite number of quark loops. It is widely used to describe meson properties in vacuum and hot medium [30]. However, in the presence of electromagnetic fields, the treatment of the Schwinger phase in the summation of quark loops for charged mesons becomes difficult. The problem comes from the lack of translation invariance of the Schwinger phase in the quark propagator. For neutral

Published by the American Physical Society under the terms of the Creative Commons Attribution 4.0 International license. Further distribution of this work must maintain attribution to the author(s) and the published article's title, journal citation, and DOI. Funded by SCOAP ${ }^{3}$. meson polarization functions the two Schwinger phases for the quark-antiquark pair cancel to each other, but for charged mesons one cannot simply perform a Fourier transformation to obtain polarization functions in momentum space. A direct way to deal with the problem is to work in coordinate space all the way, but the calculation is expected to be of great complexity. In some of the calculations, people simply neglect the Schwinger phase and take only the invariant part $[31,32]$. In the derivative expansion approach, only one quark loop is taken into account, and it can be shown to be the low-momentum approximation of the RPA approach (see Sec. IV). It is clear that the derivative expansion is reliable for light mesons, especially for pions which are the pseudo-Goldstone modes corresponding to chiral symmetry breaking.

In this paper, we study meson properties in magnetized quark matter in the frame of a two-flavor Nambu-JonaLasinio model. After a simple introduction of the mean field calculation in the model in Sec. II, we focus on deriving effective meson Lagrangian density with minimal coupling to electromagnetic fields, by taking derivative expansion for both the meson fields and Schwinger phases in Sec. III. We extract from the meson Lagrangian density the meson masses and wave function renormalization constants in this section. We show numerical calculations for the meson curvature, pole and screening masses at finite temperature and magnetic field in Sec. IV. We summarize in Sec. V.

\section{MEAN FIELD APPROXIMATION}

Nambu-Jona-Lasinio (NJL) models at the quark level describe well the chiral symmetry breaking in vacuum and its restoration at finite temperature and baryon density $[30,33,34]$. In the models, mesons are treated as collective 
excitations of quarks, and the magnetic effect on mesons comes from the magnetized quarks. In the presence of an external electromagnetic field the two-flavor NJL model is defined as

$$
\mathcal{L}=\bar{\psi}\left(i \gamma^{\mu} D_{\mu}-m_{0}\right) \psi+G\left[(\bar{\psi} \psi)^{2}+\left(\bar{\psi} i \gamma_{5} \tau \psi\right)^{2}\right],
$$

where $D_{\mu}=\partial_{\mu}+i Q A_{\mu}$ is the covariant derivative with the electromagnetic potential $A_{\mu}=(0,0, B x, 0)$ which gives a constant magnetic field along the $z$ axis $\mathbf{B}=B \mathbf{e}_{z}, Q=$ $\operatorname{diag}\left(Q_{u}=2 e / 3, Q_{d}=-e / 3\right)$ the diagonal quark charge matrix in flavor space, $m_{0}$ the current quark mass characterizing the explicit chiral symmetry breaking, $\tau$ the Pauli matrices in isospin space, and $G$ the quark coupling constant in scalar and pseudoscalar channels.

Introducing scalar and pseudoscalar meson fields $M(x)=(\sigma(x), \boldsymbol{\pi}(x))=\left(-2 G \bar{\psi}(x) \psi(x),-2 G \bar{\psi}(x) i \gamma^{5} \tau \psi(x)\right)$, and integrating out the fermion fields, the effective Lagrangian density of the model is expressed as

$$
\mathcal{L}=-\sum_{M} \frac{\left(g_{M} M\right)^{2}}{4 G}-i \operatorname{Tr} \ln \left(i \gamma^{\mu} D_{\mu}-m_{0}-K\right),
$$

where $K=\sum_{M} \Gamma_{M} M$ shows the interaction between mesons and quarks, $\Gamma_{M}=\left(\Gamma_{\sigma}, \Gamma_{\pi}\right)=\left(g_{\sigma}, i g_{\pi} \gamma_{5} \tau\right)$ are the interaction vertexes with meson coupling constants $g_{\sigma}$ and $g_{\pi}$, and $\operatorname{Tr}$ means the trace in inner spaces like color, flavor and spin.

Introducing the ensemble average $\langle\sigma\rangle$ which is the order parameter of the chiral phase transition, making a shift $\sigma(x) \rightarrow\langle\sigma\rangle+\sigma(x)$, and keeping only the condensate $\langle\sigma\rangle$ and dropping all the meson fluctuations, one obtains the mean field Lagrangian density

$$
\mathcal{L}_{\mathrm{MF}}=-i \operatorname{Tr} \ln S^{-1}-\frac{\left(m-m_{0}\right)^{2}}{4 G}
$$

and the corresponding thermodynamical potential

$$
\Omega_{\mathrm{MF}}=\frac{\left(m-m_{0}\right)^{2}}{4 G}+i \frac{T}{V} \sum_{x} \operatorname{Tr} \ln S^{-1}
$$

with the definition $\sum_{x}=\int d^{4} x \quad\left(\sum_{p}=\int d^{4} p /(2 \pi)^{4}\right)$, where $m=m_{0}+g_{\sigma}\langle\sigma\rangle$ is the dynamic quark mass, and $S=\left(i \gamma^{\mu} D_{\mu}-m\right)^{-1}=\operatorname{diag}\left(S_{u}, S_{d}\right)$ is the quark propagator in the mean field approximation.

The physical value of the chiral condensate $\langle\sigma\rangle$ or the quark mass $m$ is determined by the minimum of the thermodynamical potential $\partial \Omega_{\mathrm{MF}} / \partial\langle\sigma\rangle=0$, namely the gap equation

$$
m=m_{0}+2 i G \sum_{x} \operatorname{Tr} S=m_{0}+2 i G N_{c} \sum_{q, p} \operatorname{Tr} \tilde{S}_{q}(p),
$$

where $\tilde{S}_{q}(p)$ is the Fourier transformation of the translation invariant part $\tilde{S}_{q}(x-y)$ of the quark propagator $S_{q}(x, y)=$ $e^{i \Phi_{q}(x, y)} \tilde{S}_{q}(x-y)$ defined in Schwinger formalism [4],

$$
\begin{aligned}
\tilde{S}_{q}= & i e^{-p_{\perp}^{2} /\left|Q_{q} B\right|} \sum_{n=0}^{\infty}(-1)^{n} \frac{D_{n}(p)}{G_{n}(p)} \\
G_{n}= & p_{0}^{2}-2 n\left|Q_{q} B\right|-p_{3}^{2}-m^{2}, \\
D_{n}= & \left(p_{0} \gamma_{0}-p_{3} \gamma_{3}+m\right)\left[\left(1-i s_{q} \gamma_{1} \gamma_{2}\right) L_{n}\left(p_{q}\right)\right. \\
& \left.-\left(1+i s_{q} \gamma_{1} \gamma_{2}\right) L_{n-1}\left(p_{q}\right)\right]+4\left(p_{1} \gamma_{1}+p_{2} \gamma_{2}\right) L_{n-1}^{1}\left(p_{q}\right)
\end{aligned}
$$

with $s_{q}=\operatorname{sgn}\left(Q_{q} B\right), p_{q}=2 p_{\perp}^{2} /\left|Q_{q} B\right|$ and Laguerre polynomials $L_{n}(z)$. Note that the Schwinger phase $\Phi_{q}(x, y)$ in the quark propagator is not translation invariant.

Using the orthogonal relationship for the Laguerre polynomials and performing Matsubara frequency summation at finite temperature, the gap equation can be explicitly written as an integral over the momentum along the field direction and a summation over the Landau energy level,

$$
\begin{aligned}
& m=m_{0}+4 m G I_{2}, \\
& I_{2}=N_{c} \sum_{q, n, p_{3}} \frac{\left|Q_{q} B\right|}{2 \pi} \alpha_{n} \frac{1-2 n_{f}\left(E_{q, n}\right)}{2 E_{q, n}}
\end{aligned}
$$

with the spin degeneracy factor $\alpha_{n}=2-\delta_{n 0}$, the FermiDirac distribution $n_{f}(x)=1 /\left(1+e^{x / T}\right)$ and the quark energy $E_{q, n}=\sqrt{2 n\left|Q_{q} B\right|+p_{3}^{2}+m^{2}}$.

\section{MESON PROPERTIES FROM BOSONIZATION}

We now go beyond the mean field approximation. Including the $\sigma$ and $\pi$ fluctuations, the effective Lagrangian density (2) can be formally written as

$$
\mathcal{L}=\mathcal{L}_{\mathrm{MF}}+\mathcal{L}_{\mathrm{FD}}-\frac{2\left(m-m_{0}\right) \sigma+\sum_{M}\left(g_{M} M\right)^{2}}{4 G} .
$$

The fermionic determinant part $\mathcal{L}_{\mathrm{FD}}$ contains highly nonlocal interactions. It is desirable to localize it in a systematic fashion, and one such procedure is the derivative expansion of the fields $[4,19,28-30,32]$. To this end, we first make Taylor expansion,

$$
\begin{aligned}
& \mathcal{L}_{\mathrm{FD}}=-i \operatorname{Tr} \ln (1-S K)=\sum_{n=1}^{\infty} \mathcal{L}^{(n)}, \\
& \mathcal{L}^{(n)}=\frac{i}{n} \operatorname{Tr}(S K)^{n} .
\end{aligned}
$$

We first work out the first term $\mathcal{L}^{(1)}=i \operatorname{Tr}(S K)$, which is the linear term in the scalar meson field, 


$$
\mathcal{L}^{(1)}=i \operatorname{Tr}\left(S_{u}+S_{d}\right) g_{\sigma} \sigma=i N_{c} \sum_{q, p} \operatorname{Tr} \tilde{S}_{q}(p) g_{\sigma} \sigma .
$$

Combining with the other linear term in $\mathcal{L}$, the disappearance of the whole linear term from the effective Lagrangian recovers the gap equation (5) for the quark mass.

The meson kinetic energies and masses are extracted from the second term with $n=2$ in $\mathcal{L}_{\mathrm{FD}}, \mathcal{L}^{(2)}=i / 2 \mathrm{Tr}$ $(S K)^{2}$. The trace in flavor space gives

$$
\begin{aligned}
\operatorname{Tr}_{q}(S K)^{2}= & g_{\sigma}^{2}\left(S_{u} \sigma S_{u} \sigma+S_{d} \sigma S_{d} \sigma\right) \\
& +g_{\pi}^{2}\left(S_{u} i \gamma_{5} \pi_{0} S_{u} i \gamma_{5} \pi_{0}+S_{d} i \gamma_{5} \pi_{0} S_{d} i \gamma_{5} \pi_{0}\right) \\
& +2 g_{\pi}^{2}\left(S_{u} i \gamma_{5} \pi_{-} S_{d} i \gamma_{5} \pi_{+}+S_{d} i \gamma_{5} \pi_{+} S_{u} i \gamma_{5} \pi_{-}\right) .
\end{aligned}
$$

For the $\sigma$ part we have

$$
\begin{aligned}
\mathcal{L}_{\sigma}^{(2)}= & \frac{i g_{\sigma}^{2} N_{c}}{2} \sum_{q, y} e^{i\left(\Phi_{q}(x, y)+\Phi_{q}(y, x)\right)} \\
& \times \operatorname{Tr} \tilde{S}_{q}(x-y) \tilde{S}_{q}(y-x) \sigma(y) \sigma(x) \\
= & \frac{i g_{\sigma}^{2} N_{c}}{2} \sum_{q, y} \operatorname{Tr} \tilde{S}_{q}(x-y) \tilde{S}_{q}(y-x) \sigma(y) \sigma(x),
\end{aligned}
$$

where the two Shwinger phases cancel to each other, $\Phi_{q}(x, y)+\Phi_{q}(y, x)=0$, and only the translation invariant part of the quark propagator contributes to the action. We then take a local expansion of the $\sigma$ field at $x$,

$$
\begin{aligned}
\sigma(y)= & \sigma(x)+(y-x)^{\mu} \partial_{\mu} \sigma(x) \\
& +\frac{1}{2}(y-x)^{\mu}(y-x)^{\nu} \partial_{\mu} \partial_{\nu} \sigma(x)+\cdots .
\end{aligned}
$$

By substituting this expansion into $\mathcal{L}_{\sigma}^{(2)}$, the first term which is proportional to $\sigma^{2}(x)$ contributes to the meson curvature masses,

$$
\bar{m}_{\sigma}^{2}=-i g_{\sigma}^{2} N_{c} \sum_{q, p} \operatorname{Tr} \tilde{S}_{q}(p) \tilde{S}_{q}(p)=-2 g_{\sigma}^{2}\left(I_{2}-2 m^{2} I_{0}\right),
$$

where the integral $I_{2}$ is shown in (7) and $I_{0}$ is defined by

$$
I_{0}=N_{c} \sum_{q, n, p_{3}} \frac{\left|Q_{q} B\right|}{2 \pi} \alpha_{n} \frac{1-2 n_{f}\left(E_{q, n}\right)+2 E_{q, n} n_{f}^{\prime}\left(E_{q, n}\right)}{4 E_{q, n}^{3}}
$$

with the notation $n_{f}^{\prime}(x)=d n_{f}(x) / d x$. Note that $\bar{m}_{\sigma}$ is only a part of the curvature mass, the other quadratic term in $\mathcal{L}$ contributes to the curvature mass too.

The second term in $\mathcal{L}_{\sigma}^{(2)}$ which is linear in $(y-x)_{\mu}$ is the surface term and can be shown to vanish, and the third term $\sim \sigma(x) \partial_{\mu} \partial_{\nu} \sigma(x)$ gives the kinetic energy. Performing a Fourier transformation for the translation invariant part of the quark propagator and doing partial integration, the third term becomes

$$
\begin{aligned}
& \frac{i g_{\sigma}^{2} N_{c}}{4} \sum_{q, y} \operatorname{Tr} \tilde{S}_{q}(x-y) \tilde{S}_{q}(y-x) \\
& \quad \times \sigma(x)(y-x)^{\mu}(y-x)^{\nu} \partial_{\mu} \partial_{\nu} \sigma(x) \\
& =\frac{1}{2} \mathcal{F}_{\sigma}^{\mu \nu} \partial_{\mu} \sigma(x) \partial_{\nu} \sigma(x),
\end{aligned}
$$

with the coefficients

$$
\mathcal{F}_{\sigma}^{\mu \nu}=-\frac{i g_{\sigma}^{2} N_{c}}{2} \sum_{q, p, k, z} \operatorname{Tr} e^{i(p-k) z} z^{\mu} z^{\nu} \tilde{S}_{q}(p) \tilde{S}_{q}(k),
$$

which can be considered as wave function renormalization constants for the $\sigma$ field. Using the relations $z_{\mu} e^{i(k-p) z}=$ $i \partial_{\mu}^{p} e^{i(k-p) z}$ and $z_{\mu} z_{\nu} e^{i(k-p) z}=-\partial_{\nu}^{p} \partial_{\mu}^{p} e^{i(k-p) z}$, we have

$$
\mathcal{F}_{\sigma}^{\mu \nu}=\frac{i g_{\sigma}^{2} N_{c}}{2} \sum_{q, p} \operatorname{Tr}\left[\tilde{S}_{q}(p) \frac{\partial^{2}}{\partial p_{\mu} \partial p_{\nu}} \tilde{S}_{q}(p)\right] .
$$

The coefficients $\mathcal{F}_{\sigma}^{\mu \nu}$ with $\mu \neq \nu$ can be shown to vanish, and the nontrivial elements are only the diagonal ones $\mathcal{F}_{\sigma}^{00}$, $\mathcal{F}_{\sigma}^{33}, \mathcal{F}_{\sigma}^{11}$ and $\mathcal{F}_{\sigma}^{22}$. While the system is no longer isotropic in a background magnetic field, the coefficients in the directions perpendicular to the field should be the same, $\mathcal{F}_{\sigma}^{11}=\mathcal{F}_{\sigma}^{22}$. After taking trace in Dirac space, integrating over $p_{1}$ and $p_{2}$, and making a Wick rotation, we have the wave function renormalization constants

$$
\begin{aligned}
\mathcal{F}_{\sigma}^{00} & =\frac{g_{\sigma}^{2} N_{c}}{\pi} \sum_{q, n, p_{0}, p_{3}}\left|Q_{q} B\right| \alpha_{n} \frac{\left(p_{0}^{2}+E_{q, n}^{2}\right)^{2}-2\left(p_{0}^{2}+m^{2}\right)\left(p_{0}^{2}+E_{q, n}^{2}\right)+8 m^{2} p_{0}^{2}}{\left(p_{0}^{2}+E_{q, n}^{2}\right)^{4}} \\
\mathcal{F}_{\sigma}^{33} & =\frac{g_{\sigma}^{2} N_{c}}{\pi} \sum_{q, n, p_{0}, p_{3}}\left|Q_{q} B\right| \alpha_{n} \frac{\left(p_{0}^{2}+E_{q, n}^{2}\right)^{2}-2\left(p_{3}^{2}+m^{2}\right)\left(p_{0}^{2}+E_{q, n}^{2}\right)+8 m^{2} p_{3}^{2}}{\left(p_{0}^{2}+E_{q, n}^{2}\right)^{4}} \\
\mathcal{F}_{\sigma}^{11} & =-\frac{g_{\sigma}^{2} N_{c}}{2 \pi} \sum_{q, i, j, p_{0}, p_{3}}(-1)^{i+j} \frac{\left(p_{0}^{2}+p_{3}^{2}-m^{2}\right) J_{1}(i, j)+4\left|Q_{q} B\right| J_{2}(i, j)}{\left(p_{0}^{2}+E_{q, i}^{2}\right)\left(p_{0}^{2}+E_{q, j}^{2}\right)}
\end{aligned}
$$


where $J_{1}$ and $J_{2}$ are integrals defined as

$$
\begin{aligned}
J_{1}(i, j)= & \sum_{k=0}^{1} \int_{0}^{\infty} d u e^{-u} L_{i-k}(u)\left[(u-2) L_{j-k}(u)\right. \\
& \left.+4(u-1) L_{j-k-1}^{1}(u)+4 u L_{j-k-2}^{2}(u)\right], \\
J_{2}(i, j)= & \int_{0}^{\infty} d u e^{-u} u L_{i-1}^{1}(u)\left[(u-4) L_{j-1}^{1}(u)\right. \\
& \left.+4(u-2) L_{j-2}^{2}(u)+4 u L_{j-3}^{3}(u)\right] .
\end{aligned}
$$

From the orthogonal normalization of the Laguerre polynomials, $J_{1}$ and $J_{2}$ can be explicitly worked out,

$$
J_{1}(i, j)=-(2 i-1) \delta_{j, i-1}-(2 i+1) \delta_{j, i+1}-4 i \delta_{j, i},
$$

for $i \geq 1$,

$$
\begin{aligned}
& J_{1}(0,0)=J_{1}(0,1)=-1, \quad \text { for } i=0, \\
& J_{2}(i, j)=-i(i-1) \delta_{j, i-1}-i(i+1) \delta_{j, i+1}-2 i^{2} \delta_{j, i},
\end{aligned}
$$$$
\text { for } i \geq 1 \text {. }
$$

Note that the integral over $p_{0}$ in (19) means the Matsubara frequency summation in the imaginary time formalism of finite temperature field theory.

For the neutral pion field $\pi_{0}$, we can straightforwardly treat its contribution to $\mathcal{L}^{(2)}$,

$$
\mathcal{L}_{\pi_{0}}^{(2)}=\frac{i g_{\pi}^{2} N_{c}}{2} \sum_{q, y} \operatorname{Tr} \tilde{S}_{q}(x-y) i \gamma_{5} \tilde{S}_{q}(y-x) i \gamma_{5} \pi_{0}(y) \pi_{0}(x),
$$

by taking a similar calculation as for the $\sigma$ field, and extract its contribution to the curvature mass and the wave function renormalization constants,

$$
\begin{aligned}
\bar{m}_{\pi_{0}}^{2}= & -2 g_{\pi}^{2} I_{2}, \\
\mathcal{F}_{\pi_{0}}^{00}= & \frac{g_{\pi}^{2} N_{c}}{\pi} \sum_{q n, p_{0}, p_{3}}\left|Q_{q} B\right| \alpha_{n} \frac{E_{q, n}^{2}-p_{0}^{2}}{\left(p_{0}^{2}+E_{q, n}^{2}\right)^{3}}, \\
\mathcal{F}_{\pi_{0}}^{33}= & \frac{g_{\pi}^{2} N_{c}}{\pi} \sum_{q, n, p_{0}, p_{3}}\left|Q_{q} B\right| \alpha_{n} \frac{p_{0}^{2}+E_{q, n}^{2}-2 p_{3}^{2}}{\left(p_{0}^{2}+E_{q, n}^{2}\right)^{3}}, \\
\mathcal{F}_{\pi_{0}}^{11}= & -\frac{g_{\pi}^{2} N_{c}}{2 \pi} \sum_{q, i, j, p_{0}, p_{3}}(-1)^{i+j} \\
& \times \frac{\left(p_{0}^{2}+p_{3}^{2}+m^{2}\right) J_{1}(i, j)+4\left|Q_{q} B\right| J_{2}(i, j)}{\left(p_{0}^{2}+E_{q, i}^{2}\right)\left(p_{0}^{2}+E_{q, j}^{2}\right)} .
\end{aligned}
$$

We now calculate the charged meson part of the effective Lagrangian $\mathcal{L}^{(2)}$. Different from neutral mesons, the two Schwinger phases which appeared in the quark loop do not cancel each other and have a contribution to the Lagrangian. Taking the full quark propagator, the $\pi_{-}$part is written as

$$
\begin{aligned}
\mathcal{L}_{\pi_{-}}^{(2)}= & i g_{\pi}^{2} N_{c} \sum_{y} e^{i \Phi_{\pi_{-}}(y, x)} \operatorname{Tr} \tilde{S}_{u}(x-y) \\
& \times i \gamma^{5} \tilde{S}_{d}(y-x) i \gamma^{5} \pi_{-}(y) \pi_{+}(x)
\end{aligned}
$$

with the Schwinger phase

$$
\begin{aligned}
\Phi_{\pi_{-}}(y, x) & =\Phi_{u}(x, y)+\Phi_{d}(y, x) \\
& =Q_{u} \int_{y}^{x} A^{\mu}\left(x^{\prime}\right) d x_{\mu}^{\prime}+Q_{d} \int_{x}^{y} A^{\mu}\left(x^{\prime}\right) d x_{\mu}^{\prime} \\
& =-e \int_{x}^{y} A^{\mu}\left(x^{\prime}\right) d x_{\mu}^{\prime} .
\end{aligned}
$$

By taking a local expansion for both the charged field and the phase,

$$
\begin{aligned}
\pi_{-}(y)= & \pi_{-}(x)+(y-x)^{\mu} \partial_{\mu} \pi_{-}(x) \\
& +\frac{1}{2}(y-x)^{\mu}(y-x)^{\nu} \partial_{\mu} \partial_{\nu} \pi_{-}(x)+\cdots, \\
e^{i \Phi_{\pi_{-}}(y, x)}= & 1-i e A^{\mu}(x)(y-x)_{\mu} \\
& -i e \frac{1}{2} \frac{\partial A^{\mu}(x)}{\partial x^{\nu}}(y-x)_{\mu}(y-x)_{\nu} \\
& +\frac{(-i e)^{2}}{2}\left(A^{\mu}(x)(y-x)_{\mu}\right)^{2}+\cdots,
\end{aligned}
$$

$\mathcal{L}_{\pi_{-}}^{(2)}$ up to the quadratic term is expressed as

$$
\begin{aligned}
\mathcal{L}_{\pi_{-}}^{(2)}= & i g_{\pi}^{2} N_{c} \sum_{y} \operatorname{Tr} \tilde{S}_{u}(x-y) i \gamma^{5} \tilde{S}_{d}(y-x) i \gamma^{5} \\
& \times\left[\pi_{-}(x)+(y-x)^{\mu} D_{\mu}^{-} \pi_{-}(x)\right. \\
& \left.+\frac{1}{2}(y-x)^{\mu}(y-x)^{\nu} D_{\mu}^{-} D_{\nu}^{-} \pi_{-}(x)\right] \pi_{+}(x)
\end{aligned}
$$

with the covariant derivative $D_{\mu}^{-}=\partial_{\mu}-i e A_{\mu}(x)$. Taking almost the same calculation for the positively charged pion field, we have

$$
\begin{aligned}
\mathcal{L}_{\pi_{+}}^{(2)}= & i g_{\pi}^{2} N_{c} \sum_{y} \operatorname{Tr} \tilde{S}_{d}(x-y) i \gamma^{5} \tilde{S}_{d}(y-x) i \gamma^{5} \\
& \times\left[\pi_{+}(x)+(y-x)^{\mu} D_{\mu}^{+} \pi_{+}(x)\right. \\
& \left.+\frac{1}{2}(y-x)^{\mu}(y-x)^{\nu} D_{\mu}^{+} D_{\nu}^{+} \pi_{+}(x)\right] \pi_{-}(x)
\end{aligned}
$$

with the covariant derivative $D_{\mu}^{+}=\partial_{\mu}+i e A_{\mu}(x)$. The two terms linear in $(y-x)^{\mu}$ in $\mathcal{L}_{\pi_{-}}^{(2)}$ and $\mathcal{L}_{\pi_{+}}^{(2)}$ can be summed up to give a surface term which vanishes in the sense of coordinate integration. We finally write the $\pi_{ \pm}$contribution to the effective Lagrangian in terms of the masses $\bar{m}_{\pi_{ \pm}}$and 
the wave function renormalization constants $\mathcal{F}_{\pi_{ \pm}}^{\mu \mu}$ under the current gauge $\partial^{\mu} A_{\mu}=0$,

$$
\begin{aligned}
\mathcal{L}_{\pi_{+}}^{(2)}+\mathcal{L}_{\pi_{-}}^{(2)}= & \frac{1}{2}\left(\mathcal{F}_{\pi_{+}}^{\mu \mu}\left|D_{\mu} \pi_{+}\right|^{2}+\mathcal{F}_{\pi_{-}}^{\mu \mu}\left|D_{\mu} \pi_{-}\right|^{2}\right) \\
& -\frac{1}{2}\left(\bar{m}_{\pi_{+}}^{2}\left|\pi_{+}\right|^{2}+\bar{m}_{\pi_{-}}^{2}\left|\pi_{-}\right|^{2}\right),
\end{aligned}
$$

with the coefficients

$$
\begin{aligned}
\bar{m}_{\pi_{-}}^{2}= & -\frac{2 g_{\pi}^{2} N_{c}\left|Q_{u} B\right|}{\pi} \sum_{i, j, p_{0}, p_{3}}(-1)^{i+j} \\
& \times \frac{Y_{12}}{\left(p_{0}^{2}+E_{d, j}^{2}\right)\left(p_{0}^{2}+E_{u, i}^{2}\right)}, \\
\mathcal{F}_{\pi_{-}}^{00}= & \frac{2 g_{\pi}^{2} N_{c}\left|Q_{u} B\right|}{\pi} \sum_{i, j, p_{0}, p_{3}}(-1)^{i+j} \\
& \times \frac{Y_{12}\left(E_{u, i}^{2}-3 p_{0}^{2}\right)+2 p_{0}^{2} Y_{1}\left(p_{0}^{2}+E_{u, i}^{2}\right)}{\left(p_{0}^{2}+E_{u, i}^{2}\right)^{3}\left(p_{0}^{2}+E_{d, j}^{2}\right)}, \\
\mathcal{F}_{\pi_{-}}^{33}= & \frac{2 g_{\pi}^{2} N_{c}\left|Q_{u} B\right|}{\pi} \sum_{i, j, p_{0}, p_{3}}(-1)^{i+j} \\
& \times \frac{Y_{12}\left(p_{0}^{2}+E_{u, i}^{2}-4 p_{3}^{2}\right)+2 p_{3}^{2} Y_{1}\left(p_{0}^{2}+E_{u, i}^{2}\right)}{\left(p_{0}^{2}+E_{u, i}^{2}\right)^{3}\left(p_{0}^{2}+E_{d, j}^{2}\right)}, \\
\mathcal{F}_{\pi_{-}}^{11}= & \frac{g_{\pi}^{2} N_{c}}{\pi} \sum_{i, j, p_{0}, p_{3}}(-1)^{i+j} \frac{H_{12}}{\left(p_{0}^{2}+E_{d, j}^{2}\right)\left(p_{0}^{2}+E_{u, i}^{2}\right)},
\end{aligned}
$$

where the integrals $Y_{1}, Y_{2}, H_{1}, H_{2}, Y_{12}$ and $H_{12}$ are defined as

$$
\begin{aligned}
Y_{1}(i, j)= & \int_{0}^{\infty} d u e^{-3 u / 2}\left[L_{i}(u) L_{j-1}(2 u)+L_{i-1}(u) L_{j}(2 u)\right], \\
Y_{2}(i, j)= & \int_{0}^{\infty} d u e^{-3 u / 2} u L_{i-1}^{1}(u) L_{j-1}^{1}(2 u), \\
H_{1}(i, j)= & \sum_{k=0}^{1} \int_{0}^{\infty} d u e^{-3 u / 2} L_{j-k}(2 u)\left[(u-2) L_{i-k}(u)\right. \\
& \left.+4(u-1) L_{i-k-1}^{1}(u)+4 u L_{i-k-2}^{2}(u)\right], \\
H_{2}(i, j)= & \int_{0}^{\infty} d u e^{-3 u / 2} u L_{j-1}^{1}(2 u)\left[(u-4) L_{i-1}^{1}(u)\right. \\
& \left.+4(u-2) L_{i-2}^{2}(u)+4 u L_{i-3}^{3}(u)\right], \\
Y_{12}= & \left(p_{0}^{2}+p_{3}^{2}+m^{2}\right) Y_{1}-4\left|Q_{u} B\right| Y_{2}, \\
H_{12}= & \left(p_{0}^{2}+p_{3}^{2}+m^{2}\right) H_{1}-4\left|Q_{u} B\right| H_{2} .
\end{aligned}
$$

Due to the symmetry between the quark loops for $\pi_{+}$and $\pi_{-}$, it is easy to show $\bar{m}_{\pi_{+}}=\bar{m}_{\pi_{-}}$and $\mathcal{F}_{\pi_{+}}^{\mu \mu}=\mathcal{F}_{\pi_{-}}^{\mu \mu}$. Note that, including self-consistently, the Schwinger phases $\Phi_{\pi_{ \pm}}$ in the calculation lead to the covariant derivatives $D_{\mu}^{ \pm}$ acting on the charged pion fields, which guarantee the minimal coupling between the meson fields and the gauge field in the frame of bosonization.

At this point we should discuss the convergence of the derivative expansion for the Schwinger phase, the second line of Eq. (25). Similar to the expansion for a meson field, Eq. (13) or the first line of (25), which needs a small meson momentum to guarantee the convergence of the expansion, an effective expansion for the Schwinger phase requires small field strengths $E, B \sim \partial_{\mu} A^{\mu}$. Considering that the strongest magnetic field in nature is at the order of pion mass, $e B \sim\left((1-5) m_{\pi}\right)^{2}$, the constraint on the electromagnetic fields is not so serious as the constraint on the meson mass.

Putting together all the contributions from neutral and charged mesons $\mathcal{L}_{\sigma}^{(2)}, \mathcal{L}_{\pi_{0}}^{(2)}, \mathcal{L}_{\pi_{+}}^{(2)}$ and $\mathcal{L}_{\pi_{-}}^{(2)}$ calculated above, we obtain the effective Lagrangian of the quark-meson plasma at quasiparticle level,

$$
\begin{aligned}
\mathcal{L}= & \mathcal{L}_{\mathrm{MF}}+\sum_{M}\left[\frac{1}{2} \mathcal{F}_{M}^{\mu \mu}\left|\mathcal{D}_{\mu}^{M} M(x)\right|^{2}\right. \\
& \left.-\frac{1}{2}\left(\frac{g_{M}^{2}}{2 G}+\bar{m}_{M}^{2}\right)|M(x)|^{2}\right]
\end{aligned}
$$

with $\mathcal{D}_{\mu}^{M}=\partial_{\mu}$ for neutral mesons and $D_{\mu}^{ \pm}=\partial_{\mu} \pm i e A_{\mu}$ for charged mesons, where we have neglected the linear term in $\sigma$ and all the surface terms. The effective action (31) defines the meson curvature masses

$$
m_{M}^{2}=\frac{g_{M}^{2}}{2 G}+\bar{m}_{M}^{2}
$$

For the neutral pion, we have from (22) $m_{\pi_{0}}^{2}=g_{0}^{2} /(2 G)$ $\left(1-4 G I_{2}\right)$. The comparison with the quark gap equation (7) leads to $m_{\pi_{0}}=0$ in the chiral breaking phase in the chiral limit with $m_{0}=0$. This guarantees the Goldstone mode in finite temperature and magnetic field. Note that the original $S U_{L}(2) \times S U_{R}(2)$ chiral symmetry is broken down to $U_{L}(1) \times U_{R}(1)$ by the magnetic field $\mathbf{B}$ and the number of Goldstone modes is reduced from 3 to 1 .

From the on-shell conditions for noninteracting mesons in an external magnetic field,

$$
\begin{aligned}
& \mathcal{F}_{M}^{00} p_{0}^{2}-\mathcal{F}_{M}^{11} p_{1}^{2}-\mathcal{F}_{M}^{22} p_{2}^{2}-\mathcal{F}_{M}^{33} p_{3}^{2}=m_{M}^{2}, \\
& \quad \text { for } M=\sigma, \pi_{0}, \\
& \mathcal{F}_{M}^{00} p_{0}^{2}-\mathcal{F}_{M}^{11}(2 n+1)|e B|-\mathcal{F}_{M}^{33} p_{3}^{2}=m_{M}^{2} \\
& \quad \text { for } M=\pi_{+}, \pi_{-},
\end{aligned}
$$

we obtain the meson pole masses defined as $p_{0}=m_{M}^{(0)}$ at zero momentum $p_{1}=p_{2}=p_{3}=0$ for neutral mesons and at lowest Landau level and zero momentum $n=p_{3}=0$ for charged mesons, 


$$
\begin{aligned}
& m_{M}^{(0)}=m_{M} / \sqrt{\mathcal{F}_{M}^{00}} \text { for } M=\sigma, \pi_{0}, \\
& m_{M}^{(0)}=\sqrt{\left(m_{M}^{2}+\mathcal{F}_{M}^{11}|e B|\right) / \mathcal{F}_{M}^{00}} \text { for } M=\pi_{+}, \pi_{-} .
\end{aligned}
$$

We can also define the screening masses at $p_{j}=i m_{M}^{(j)}$ in the direction $\mathbf{e}_{j}$. For instance, we have for neutral mesons,

$$
m_{M}^{(j)}=m_{M} / \sqrt{\mathcal{F}_{M}^{j j}} \quad \text { for } M=\sigma, \pi_{0} .
$$

Since the two directions perpendicular to the magnetic field are symmetric, we have only two independent screening masses, the parallel and perpendicular screening masses $m_{M}^{(z)}$ and $m_{M}^{(x)}$. By considering the interaction potential between two quarks which is just a Fourier transform of the meson propagator, the screening mass $m_{M}^{(j)}$ controls the interaction range, its inverse defines the screening radius,

$$
r_{M}^{(j)}=1 / m_{M}^{(j)}
$$

It is clear that heavy mesons like the Higgs mode $\sigma$ propagate short range interaction and light mesons like the Goldstone mode $\pi_{0}$ propagate the long range interaction.

\section{NUMERICAL RESULTS}

Before we do numerical calculation in the finite temperature and magnetic field, we first fix the model parameters by fitting the meson properties in vacuum. Since temperature and magnetic field disappear in vacuum, there is no difference among the three pions $\left(m_{\pi}=m_{\pi_{0}}=m_{\pi_{ \pm}}\right)$, and the symmetry in space-time is recovered $\left(\mathcal{F}_{M}=\mathcal{F}_{M}^{00}=\right.$ $\left.\mathcal{F}_{M}^{33}=\mathcal{F}_{M}^{11}=\mathcal{F}_{M}^{22}\right)$. Taking into account the gap equation for the quark mass $m$ (7) and the pion decay constant $f_{\pi}$ in the model [30] $f_{\pi}^{2}=m^{2} I_{0}$, the meson curvature masses (32) can be expressed in terms of $m$ and $f_{\pi}$,

$$
\begin{aligned}
& m_{\pi}^{2}=\frac{g_{\pi}^{2}}{2 G} \frac{m_{0}}{m}, \\
& m_{\sigma}^{2}=\frac{g_{\sigma}^{2}}{2 G}\left(\frac{m_{0}}{m}+8 G f_{\pi}^{2}\right) .
\end{aligned}
$$

On the other hand, the meson wave function renormalization constants to the leading order are greatly simplified in vacuum,

$$
\begin{aligned}
& \mathcal{F}_{\sigma} \simeq g_{\sigma}^{2} I_{0}=g_{\sigma}^{2} \frac{f_{\pi}^{2}}{m^{2}}, \\
& \mathcal{F}_{\pi} \simeq g_{\pi}^{2} I_{0}=g_{\pi}^{2} \frac{f_{\pi}^{2}}{m^{2}} .
\end{aligned}
$$

With the Goldberger-Treiman relation [30] $f_{\pi}^{2} g_{\pi}^{2}=m^{2}$, we have

$$
\mathcal{F}_{\sigma}=\mathcal{F}_{\pi}=1
$$

in vacuum. This means that the wave function renormalization is not important in vacuum and we can safely neglect it. In this case, we have the meson pole masses

$$
m_{M}^{(0)} \simeq m_{M}^{(z)} \simeq m_{M}^{(x)} \simeq m_{M}
$$

As a nonrenormalizable theory, we need a regularization to remove the divergences in the momentum integrations in the NJL model. There are different regularization approaches, like soft cutoff [15,19], 3-momentum cutoff [16,17] and Pauli-Villars approach [18]. A high order PauliVillars regularization has two advantages. First, it allows for a larger energy scale which makes the calculation more reliable at larger external parameters like temperature, density and electromagnetic fields. Second, the transverse velocity of the Goldstone mode becomes larger than the speed of light in the soft cutoff scheme which violates the law of causality, but this trouble can be cured in PauliVillars regularization schemes [18]. Under the Pauli-Villars regularization, one replaces any integrated function $G(m)$ by $\sum_{i} c_{i} G\left(m_{i}\right)$ with regularized quark masses $m_{i}=$ $\sqrt{m^{2}+a_{i} \Lambda^{2}}$ for $i=0,1, \ldots, N-1$. The coefficients $a_{i}$ and $c_{i}$ are determined by constraints $a_{0}=0, c_{0}=1$ and $\sum_{i=0}^{N-1} c_{i} m_{i}^{2 j}=0$ for $j=0,1, \ldots, N-1$. In our treatment we take a $N=8$ Pauli-Villars regularization with $a_{i}=$ $\{0,1,2,3,4,5,6,7\}$ and $c_{i}=\{1,-7,21,-35,35,-21$, $7,-1\}$. The parameters $\Lambda=1.505 \mathrm{GeV}, G=3.44(\mathrm{GeV})^{-2}$ and $m_{0}=5.3 \mathrm{MeV}$ are obtained by fitting $f_{\pi}=93 \mathrm{MeV}$, $\langle\bar{\psi} \psi\rangle=(-246 \mathrm{MeV})^{3}$ and $m_{\pi}=135 \mathrm{MeV}$. Note that in the Pauli-Villars regularization scheme the summation over Landau energy level converges slower than that in hard and soft cutoff schemes. In our calculation the summation is performed up to $n=2000$ for $e B=2 m_{\pi}^{2}$, to guarantee the convergence of the calculation.

We now illustrate in vacuum the similarity and difference between derivative expansion and RPA approaches. In RPA, the pion and sigma meson masses are determined by the self-consistent equations [30]

$$
\begin{aligned}
& m_{\pi}^{2}=\frac{m_{0}}{m} \frac{1}{2 G \tilde{I}_{0}\left(m_{\pi}^{2}\right)}, \\
& m_{\sigma}^{2}=\frac{m_{0}}{m} \frac{1}{2 G \tilde{I}_{0}\left(m_{\sigma}^{2}\right)}+4 m^{2}
\end{aligned}
$$

with the function

$$
\begin{aligned}
\tilde{I}_{0}\left(k^{2}\right)= & -2 N_{c} N_{f} \\
& \times \int \frac{d^{4} p}{(2 \pi)^{4}} \frac{1}{\left((p-k / 2)^{2}-m^{2}\right)\left((p+k / 2)^{2}-m^{2}\right)},
\end{aligned}
$$




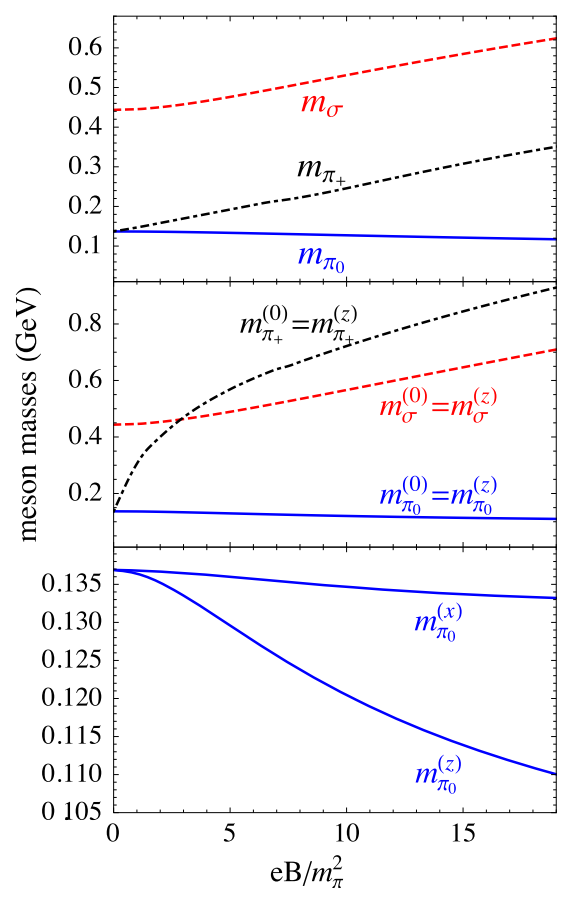

FIG. 1. The meson curvature masses (upper panel) and pole and screening masses (middle and lower panels) at zero temperature but finite magnetic field. $m_{\pi}$ is the pion mass in vacuum.

where $k$ is the meson 4-momentum. The integral $\tilde{I}_{0}$ is a smooth function of $k^{2}$, and one can do local expansion around $k^{2}=0$,

$$
\tilde{I}_{0}\left(k^{2}\right)=I_{0}+\mathcal{O}\left(k^{2}\right)
$$

Taking the low momentum limit $k^{2} \rightarrow 0$, the meson masses (41) in RPA are reduced to (37) in derivative expansion, by taking into account the meson decay constant and the Goldberger-Treiman relation.

We first show in Fig. 1 the meson curvature, pole and screening masses as functions of magnetic field at zero temperature. Considering the Lorentz invariance between the time direction and magnetic field direction at zero temperature, there is no difference between the wave function renormalizations in these two directions. Therefore, the pole mass $m_{M}^{(0)}$ is exactly the same as the parallel screening mass $m_{M}^{(z)}$. In the beginning at zero magnetic field, the difference between the parallel and perpendicular directions disappears too, and the wave function renormalization can be approximately neglected. In this case, the only mass difference is between the isospin singlet and triplet, the degenerated pion mass starts at $135 \mathrm{MeV}$ and sigma mass at $440 \mathrm{MeV}$. The nonzero magnetic field brings a mass splitting between neutral and charged pions: The curvature masses $m_{\pi_{+}}=m_{\pi_{-}}$goes up but $m_{\pi_{0}}$ goes down with increasing $e B$. For neutral mesons, the correction from wave function renormalizations in the time and $z$ directions is still weak when the

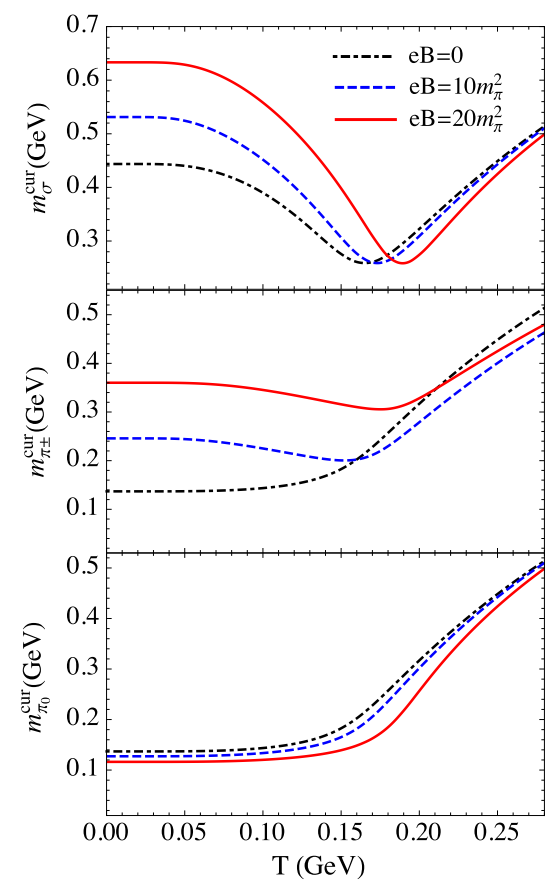

FIG. 2. The curvature masses at finite temperature and magnetic field for sigma (upper panel), charged pions (middle panel) and neutral pion (lower panel).

magnetic field is not very strong, which leads to almost the same curvature, pole and screening masses $m_{\sigma}^{(0)}=m_{\sigma}^{(z)} \simeq$ $m_{\sigma}$ and $m_{\pi_{0}}^{(0)}=m_{\pi_{0}}^{(z)} \simeq m_{\pi_{0}}$. For charged pions, however, the contribution from the lowest Landau level to the pole masses, namely the term $\mathcal{F}_{\pi_{ \pm}}^{11}|e B|$ in (34), leads to a rather strong magnetic field dependence of $m_{\pi_{ \pm}}^{(0)}=m_{\pi_{ \pm}}^{(z)}$. Taking into account the fact that $\pi_{0}$ is the lightest meson in the model and controls the interaction range between two quarks, we only consider its screening mass. In comparison with sigma and charged pions, the magnetic field dependence of the Goldstone mode is very weak, especially for its screening mass in the perpendicular direction.

We now focus on the temperature dependence of the meson curvature masses which dominate the pole and screening masses. Figure 2 shows $m_{\sigma}, m_{\pi_{ \pm}}$and $m_{\pi_{0}}$ as functions of temperature at fixed magnetic field $e B / m_{\pi}^{2}=0$, 10 and 20. At low temperature, different from $\sigma$ and charged pions $\pi_{ \pm}$which obtain mass from the interaction with magnetic field, the Goldstone mode, namely the neutral pion $\pi_{0}$, loses mass in the interaction. This means that the Goldstone mode controls the thermodynamics of the system and its importance increases with magnetic field. For the scalar meson $\sigma$ which is the Higgs mode corresponding to the chiral symmetry breaking, its mass continuously drops down at low temperature, reaches the minimum at the critical point of chiral phase transition, and then goes up in the phase with chiral symmetry restoration. At $B=0$ the critical temperature is $165 \mathrm{MeV}$. At high temperature, the magnetic field dependence of any curvature mass becomes 


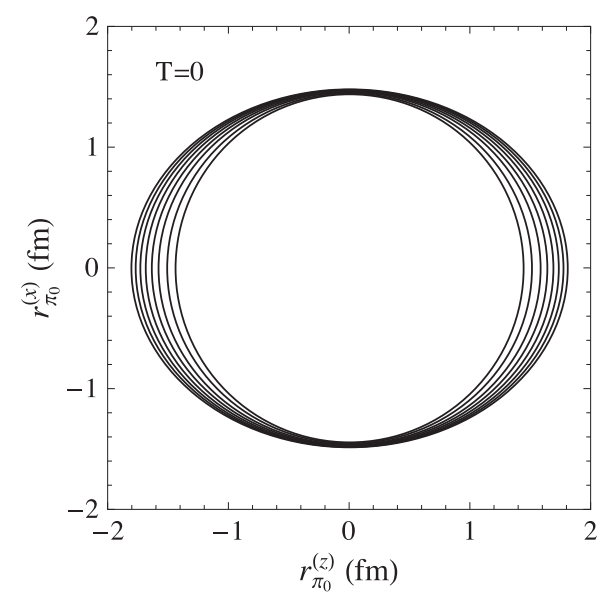

FIG. 3. The screening region of quark interaction in $z-x$ plane at zero temperature. $z$ and $x$ are the directions parallel and perpendicular to the magnetic field, and the fixed field strength corresponding to each ellipse increases from inside $\left(e B / m_{\pi}^{2}=0\right)$ to outside (20).

weak, they approach the same limit governed by the restored chiral symmetry.

The Goldstone mode controls not only the thermodynamics of the system but also the quark interaction propagated by mesons. As the lightest meson, $\pi_{0}$ governs the interaction range. The screening at finite temperature suppresses the long range part and remains only the short range part, the critical length is called screening radius determined by the screening mass, $r_{\pi_{0}}=1 / m_{\pi_{0}}$. When the symmetry in coordinate space is broken by the magnetic field, the screening radius in the directions parallel and perpendicular to the field become different, and the interaction region changes from a sphere to a ellipsoid. From $m_{\pi_{0}}^{(x)}>m_{\pi_{0}}^{(z)}$ shown in the lower panel of Fig. 1, the short axis of the ellipsoid is in the perpendicular plane $(x-y$ plane) and the long axis is in the parallel $(z)$ direction. Figure 3 shows the magnetic field dependence of the ellipse in the $z-x$ plane at zero temperature. Since $m_{\pi_{0}}^{(x)}$ is almost a constant in the magnetic field, the short axis does not change. However, with increasing magnetic field the decreased screening mass $m_{\pi_{0}}^{(z)}$ leads to an increasing long axis. At finite temperature, the random thermal motion tends to recover the space symmetry, and the ellipsoid approaches back to the sphere.

Finally we discuss the feedback from mesons to quarks. Due to the fermion dimension reduction, see the quark propagator (6), a magnetic catalysis effect on chiral symmetry breaking is expected in both vacuum and finite temperature in almost all model calculations at the mean field level [4-6]. To understand the inverse magnetic catalysis effect found in recent lattice QCD simulations with a physical pion mass [7-9], many scenarios beyond mean field approximation are proposed [10-14], such as magnetic inhibition of mesons, mass gap in the large $N_{c}$ limit, sphalerons, gluon screening effect, and weakening of strong coupling. In our calculation, quarks are treated at the mean field level and mesons are introduced as quantum fluctuations above the mean field through the derivative expansion. Since we did not consider the feedback from mesons to quarks, the mean field thermodynamic potential gives the magnetic catalysis effect. When the meson contribution to the thermodynamics of the system is included, $\Omega=\Omega_{\mathrm{MF}}+\Omega_{\mathrm{M}}$, the inverse magnetic catalysis effect is expected to be recovered, see for instance the work in Ref. [14] where the RPA approach is used to construct mesons.

\section{CONCLUSION}

In this paper, we systematically study the neutral and charged meson properties starting with a quark model at finite temperature and magnetic field. Taking the bosonization method in a two-flavor NJL model, we derive the kinetic part of the local meson Lagrangian from the nonlocal one-loop Lagrangian in the model by derivative expansion. The Schwinger phases in quark propagators caused by the magnetic potential are processed in a selfconsistent way, leading to covariant derivatives acting on charged mesons. From the kinetic part of the effective meson Lagrangian, we extract the meson curvature masses and wave function renormalization constants which together control the meson pole and screening masses.

The magnetic field breaks down the isospin symmetry among the neutral and charged pions and the symmetry in coordinate space. As a result, the pion mass splits into two branches in the magnetic field, and the wave function renormalizations in the directions parallel and perpendicular to the field are different. Due to the contribution from the lowest Landau level, the charged pions become much heavier in the magnetic field and are sensitive to the field strength. As the only Goldstone mode corresponding to spontaneous chiral symmetry breaking, the neutral pion governs the thermodynamics of the system and propagates the long range quark interaction. From the magnetic field dependence of the neutral pion screening mass, the quark interaction region changes from a sphere in vacuum to a ellipsoid in the magnetic field, and the asymmetry increases with the field strength.

\section{ACKNOWLEDGMENTS}

The work is supported by the NSFC and MOST Grants No. 11335005, No. 11575093 and No. 2014CB845400. 
[1] D. E. Kharzeev, L. D. McLerran, and H. J. Warringa, The effects of topological charge change in heavy ion collisions: Event by event $\mathrm{P}$ and $C P$ violation, Nucl. Phys. A803, 227 (2008).

[2] V. Skokov, A. Y. Illarionov, and V. Toneev, Estimate of the magnetic field strength in heavy-ion collisions, Int. J. Mod. Phys. A 24, 5925 (2009).

[3] V. Voronyuk, V. D. Toneev, W. Cassing, E. L. Bratkovskaya, V. P. Konchakovski, and S. A. Voloshin, (Electro)magnetic field evolution in relativistic heavy-ion collisions, Phys. Rev. C 83, 054911 (2011).

[4] V. P. Gusynin, V. A. Miransky, and I. A. Shovkovy, Dimensional reduction and catalysis of dynamical symmetry breaking by a magnetic field, Nucl. Phys. B462, 249 (1996).

[5] K. G. Klimenko, Three-dimensional Gross-Neveu model at nonzero temperature and in an external magnetic field, Z. Phys. C 54, 323 (1992).

[6] S. P. Klevansky and R. H. Lemmer, Chiral symmetry restoration in the Nambu-Jona-Lasinio model with a constant electromagnetic field, Phys. Rev. D 39, 3478 (1989).

[7] G. S. Bali, F. Bruckmann, G. Endrodi, Z. Fodor, S. D. Katz, S. Krieg, A. Schafer, and K. K. Szabo, The QCD phase diagram for external magnetic fields, J. High Energy Phys. 02 (2012) 044.

[8] G. S. Bali, F. Bruckmann, G. Endrodi, Z. Fodor, S. D. Katz, and A. Schafer, QCD quark condensate in external magnetic fields, Phys. Rev. D 86, 071502 (2012).

[9] G. S. Bali, F. Bruckmann, G. Endrodi, F. Gruber, and A. Schaefer, Magnetic field-induced gluonic (inverse) catalysis and pressure (an)isotropy in QCD, J. High Energy Phys. 04 (2013) 130.

[10] K. Fukushima and Y. Hidaka, Magnetic Catalysis Versus Magnetic Inhibition, Phys. Rev. Lett. 110, 031601 (2013).

[11] J. Chao, P. Chu, and M. Huang, Inverse magnetic catalysis induced by sphalerons, Phys. Rev. D 88, 054009 (2013).

[12] F. Bruckmann, G. Endrodi, and T. G. Kovacs, Inverse magnetic catalysis and the Polyakov loop, J. High Energy Phys. 04 (2013) 112.

[13] J. O. Andersen, W. R. Naylor, and A. Tranberg, Inverse magnetic catalysis and regularization in the quark-meson model, J. High Energy Phys. 02 (2015) 042.

[14] S. Mao, Inverse magnetic catalysis in Nambu-Jona-Lasinio model beyond mean field, Phys. Lett. B 758, 195 (2016).

[15] S. Fayazbakhsh and N. Sadooghi, Weak decay constant of neutral pions in a hot and magnetized quark matter, Phys. Rev. D 88, 065030 (2013).

[16] S. S. Avancini, W. R. Tavares, and M. B. Pinto, Properties of magnetized neutral mesons within a full RPA evaluation, Phys. Rev. D 93, 014010 (2016).
[17] S. S. Avancini, R. L.S. Farias, M. Benghi Pinto, W. R. Tavares, and V.S. Timteo, $\pi_{0}$ pole mass calculation in a strong magnetic field and lattice constraints, Phys. Lett. B 767, 247 (2017).

[18] S. Mao and Y. Wang, Effect of discrete quark momenta on the Goldstone mode in a magnetic field, Phys. Rev. D 96, 034004 (2017).

[19] S. Fayazbakhsh, S. Sadeghian, and N. Sadooghi, Properties of neutral mesons in a hot and magnetized quark matter, Phys. Rev. D 86, 085042 (2012).

[20] K. Kamikado and T. Kanazawa, Chiral dynamics in a magnetic field from the functional renormalization group, J. High Energy Phys. 03 (2014) 009.

[21] J. O. Andersen, Thermal pions in a magnetic background, Phys. Rev. D 86, 025020 (2012).

[22] J. O. Andersen, Chiral perturbation theory in a magnetic background-finite-temperature effects, J. High Energy Phys. 10 (2012) 005.

[23] G. Colucci, E. S. Fraga, and A. Sedrakian, Chiral pions in a magnetic background, Phys. Lett. B 728, 19 (2014).

[24] K. Hattori, T. Kojo, and N. Su, Mesons in strong magnetic fields: (I) General analyses, Nucl. Phys. A951, 1 (2016).

[25] A. Mukherjee, S. Ghosh, M. Mandal, P. Roy, and S. Sarkar, Mass modification of hot pions in a magnetized dense medium, Phys. Rev. D 96, 016024 (2017).

[26] P. Chakraborty, Meson spectral function and screening masses in magnetized quark gluon plasma, arXiv:1711 .04404

[27] S. P. Adhya, M. Mandal, S. Biswas, and P. K. Roy, Pionic dispersion relations in the presence of a weak magnetic field, Phys. Rev. D 93, 074033 (2016).

[28] T. Eguchi, A new approach to collective phenomena in superconductivity models, Phys. Rev. D 14, 2755 (1976).

[29] S. Imai, H. Toki, and W. Weise, Quark-hadron matter at finite temperature and density in a two-color PNJL model, Nucl. Phys. A913, 71 (2013).

[30] S. P. Klevansky, The Nambu-Jona-Lasinio model of quantum chromodynamics, Rev. Mod. Phys. 64, 649 (1992).

[31] H. Liu, L. Yu, M. Chernodub, and M. Huang, Possible formation of high temperature superconductor at an early stage of heavy-ion collisions, Phys. Rev. D 94, 113006 (2016).

[32] R. Zhang, W. j. Fu, and Y.x. Liu, Properties of mesons in a strong magnetic field, Eur. Phys. J. C 76, 307 (2016).

[33] Y. Nambu and G. Jona-Lasinio, Dynamical model of elementary particles based on an analogy with superconductivity. 1., Phys. Rev. 122, 345 (1961).

[34] T. Hatsuda and T. Kunihiro, QCD phenomenology based on a chiral effective Lagrangian, Phys. Rep. 247, 221 (1994). 